\title{
Variable levels of drift in tunicate cardiopharyngeal gene regulatory elements
}

\author{
William Colgan ${ }^{1}$, Alexis Leanza ${ }^{3}$, Ariel Hwang ${ }^{4}$, Melissa B. DeBiasse ${ }^{5}$, Isabel Llosa², Daniel Rodrigues², \\ Hriju Adhikari², Guillermo Barreto Corona², Saskia Bock², Amanda Carillo-Perez², Meagan Currie², \\ Simone Darkoa-Larbi ${ }^{6}$, Daniel Dellal ${ }^{7}$, Hanna Gutow ${ }^{2}$, Pascha Hokama ${ }^{8}$, Emily Kibby ${ }^{9}$, Noah Linhart ${ }^{10}$, \\ Sophia Moody ${ }^{11}$, Allison Naganuma ${ }^{2}$, Diep Nguyen ${ }^{12}$, Ryan Stanton ${ }^{2}$, Sierra Stark ${ }^{13}$, Cameron Tumey ${ }^{2}$, \\ Anthony Velleca ${ }^{14}$, Joseph F. Ryan ${ }^{5}$ and Brad Davidson ${ }^{2 *}$ (D)
}

\begin{abstract}
Background: Mutations in gene regulatory networks often lead to genetic divergence without impacting gene expression or developmental patterning. The rules governing this process of developmental systems drift, including the variable impact of selective constraints on different nodes in a gene regulatory network, remain poorly delineated.

Results: Here we examine developmental systems drift within the cardiopharyngeal gene regulatory networks of two tunicate species, Corella inflata and Ciona robusta. Cross-species analysis of regulatory elements suggests that trans-regulatory architecture is largely conserved between these highly divergent species. In contrast, cis-regulatory elements within this network exhibit distinct levels of conservation. In particular, while most of the regulatory elements we analyzed showed extensive rearrangements of functional binding sites, the enhancer for the cardiopharyngeal transcription factor FoxF is remarkably well-conserved. Even minor alterations in spacing between binding sites lead to loss of FoxF enhancer function, suggesting that bound trans-factors form position-dependent complexes.

Conclusions: Our findings reveal heterogeneous levels of divergence across cardiopharyngeal cis-regulatory elements. These distinct levels of divergence presumably reflect constraints that are not clearly associated with gene function or position within the regulatory network. Thus, levels of cis-regulatory divergence or drift appear to be governed by distinct structural constraints that will be difficult to predict based on network architecture.
\end{abstract}

Keywords: Gene regulatory networks, Developmental systems drift, Tunicates, Heart development, Selective constraints

\section{Background}

The gene regulatory networks (GRNs) that orchestrate development are largely composed of trans-regulatory factors (i.e., transcription factors) and cis-regulatory elements (i.e., enhancers and silencers) [1]. Connections within these networks are dictated by transcription factor binding sites within each regulatory element [1-3]. Mutations that alter binding site composition are a major driver of developmental changes underlying evolutionary

*Correspondence: bdavids1@swarthmore.edu

${ }^{2}$ Swarthmore College, Swarthmore, USA

Full list of author information is available at the end of the article shifts in phenotype [4-9]. However, mutations can accumulate in cis-regulatory elements without altering gene network function, contributing to developmental systems drift [10-12]. Drift can also occur in trans due to mutations that impact the expression or coding sequence of upstream transcription factors (as defined in relation to a specific target gene) [5]. In general, the organization of binding motifs within cis-regulatory elements is loosely constrained. This structural flexibility presumably reflects independent, non-cooperative binding of upstream transcription factors $[3,13,14]$. However, within a limited subset of regulatory elements, the binding site organization is more tightly constrained. This structural rigidity 
presumably reflects cooperative, position-specific interactions between bound transcription factors and associated co-factors [14-19]. The prevalence and nature of such cooperative binding interactions and the resulting impact on drift are outstanding questions in evolutionary developmental biology [3].

Although developmental systems drift in GRNs appears to be a common phenomenon in metazoan evolution, it can be difficult to study due to the requirement for rigorous cross-species analysis within well-characterized networks [11, 12, 20, 21]. Cross-species assays are used to determine the intelligibility of characterized cisregulatory elements between two species and thus evaluate hypotheses regarding the amount of drift. Mutual intelligibility of a cis-regulatory element suggests that only cis drift has occurred [22-24]. In contrast, partial or complete loss of intelligibility indicates that trans drift has occurred [10, 25, 26]. It should be noted that results from cross-species analysis are not definitive. Alterations in GRN structure may be associated with shifts in temporal or spatial expression that are difficult to detect either because they are subtle or because available techniques (such as reporter assays) do not accurately reflect endogenous expression. Thus, in general, experimental evidence for developmental systems drift does not rule out a role for selection in driving observed shifts in GRN architecture.

Tunicates, or urochordates, are a powerful system for studying developmental systems drift (Fig. 1). They are closely related to vertebrates but diverged prior to vertebrate genome duplications, so they have a single copy of many important developmental genes [27, 28]. Tunicates also have relatively compact genomes, enabling easy identification of cis-regulatory elements through phylogenetic footprinting or detection of clustered binding motifs [29-32]. In addition, some tunicate species can be electroporated en masse, enabling high-throughput testing of cis-regulatory elements with transgenic reporters [33]. These techniques have been successfully employed to intensively characterize developmental gene regulatory networks in Ciona robusta (formerly known as Ciona intestinalis, type A), including the network underlying heart and pharyngeal development (Fig. 1a-c). Furthermore, tunicate embryos employ similar, deeply conserved patterning mechanisms for early development. Remarkably, species in two major tunicate clades, Phlebobranchia and Stolidobranchia, have nearly identical embryonic fate maps and employ similar programs for specification and morphogenesis, despite having diverged $\sim 390$ million years ago (Fig. 1d) [10, 34-36]. These similarities in developmental patterning are even more striking when the extreme rate of genomic divergence between tunicate species is taken into consideration [37-40]. The unique combination of stringent developmental conservation and extreme genomic divergence makes the tunicates a powerful model for revealing the constraints that shape adaptation and developmental systems drift [37].

Previous studies of tunicate developmental systems drift have focused on comparisons to the relatively wellcharacterized regulatory networks underlying embryonic development in C. robusta [44]. For some genes, including the key developmental transcription factor Otx, conservation of the trans-regulatory environment promotes conserved expression patterns and mutual intelligibility in cross-species analysis despite extensive binding site rearrangements within cis-regulatory elements [24, 45]. In other cases, expression is conserved despite divergence of the trans-regulatory factors and associated cis-regulatory elements, leading to loss of cross-species intelligibility [26]. Drift in trans-factors is also indicated by species-specific deployment of distinct signaling pathways in otherwise conserved developmental programs, including the program driving muscle progenitor lineage induction $[46,47]$. These findings align with the hypothesis that the extreme genomic divergence between tunicate species has resulted in profound levels of drift within developmental GRNs [37].

Extensive characterization of the C. robusta cardiopharyngeal GRN makes it an attractive model for comparative studies examining developmental systems drift (Fig. 1a-c) [42, 48, 49]. The heart in C. robusta can be traced back to two blastomeres (the B7.5 cells, also termed cardiopharyngeal founder cells) which express the bHLH transcription factor Mesp (Fig. 1a) [50-52]. Founder cell-specific expression of Mesp is mediated by two upstream transcription factors: a T-Box family transcription factor, TBX6b, and a LIM homeobox family transcription factor, LHX3, which are expressed in overlapping maternally specified domains [51, 53, 54]. During gastrulation, the founder cells divide once, forming a pair of cells on each side of the embryo, and express the transcription factor Ets1/2 (Fig. 1a). The four resulting cells then divide asymmetrically, creating two distinct cell lineages: the anterior tail muscle cells (ATMs) and the trunk ventral cells (TVCs). The TVCs are bi-potential progenitors, giving rise to pharyngeal muscle and cardiac lineages (Fig. 1b). TVC specification is dictated by fibroblast growth factor (FGF)/Map Kinase (MapK)-dependent activation of Ets1/2 [55-57]. Ets1/2 in conjunction with an unknown ATTA-binding co-factor then upregulates a set of 218 primary genes which include the conserved cardiac transcription factors FoxF, Hand-like, and GATAa (Fig. 1b) $[41,58,59]$. These three transcription factors are thought to regulate distinct modules in the C. robusta cardiopharyngeal GRN (Fig. 1c) [42, 60-63]. 


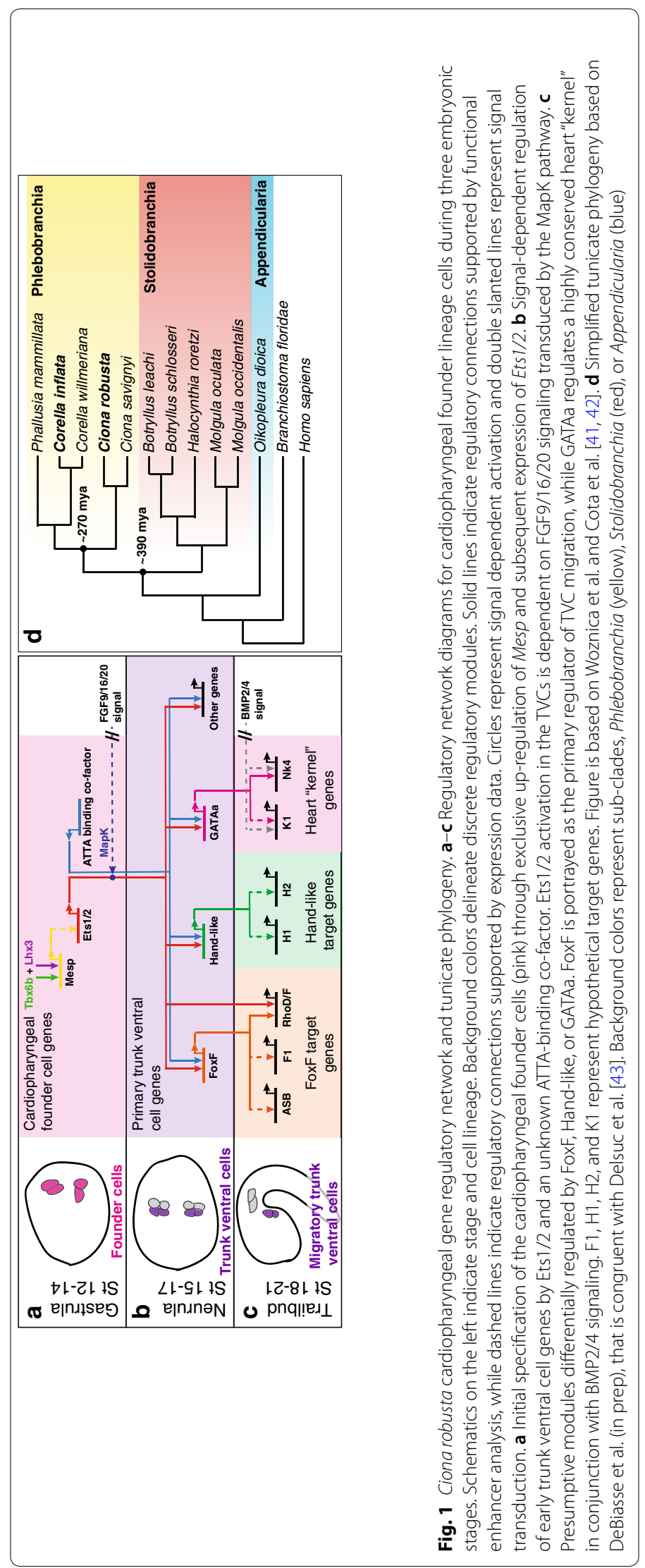



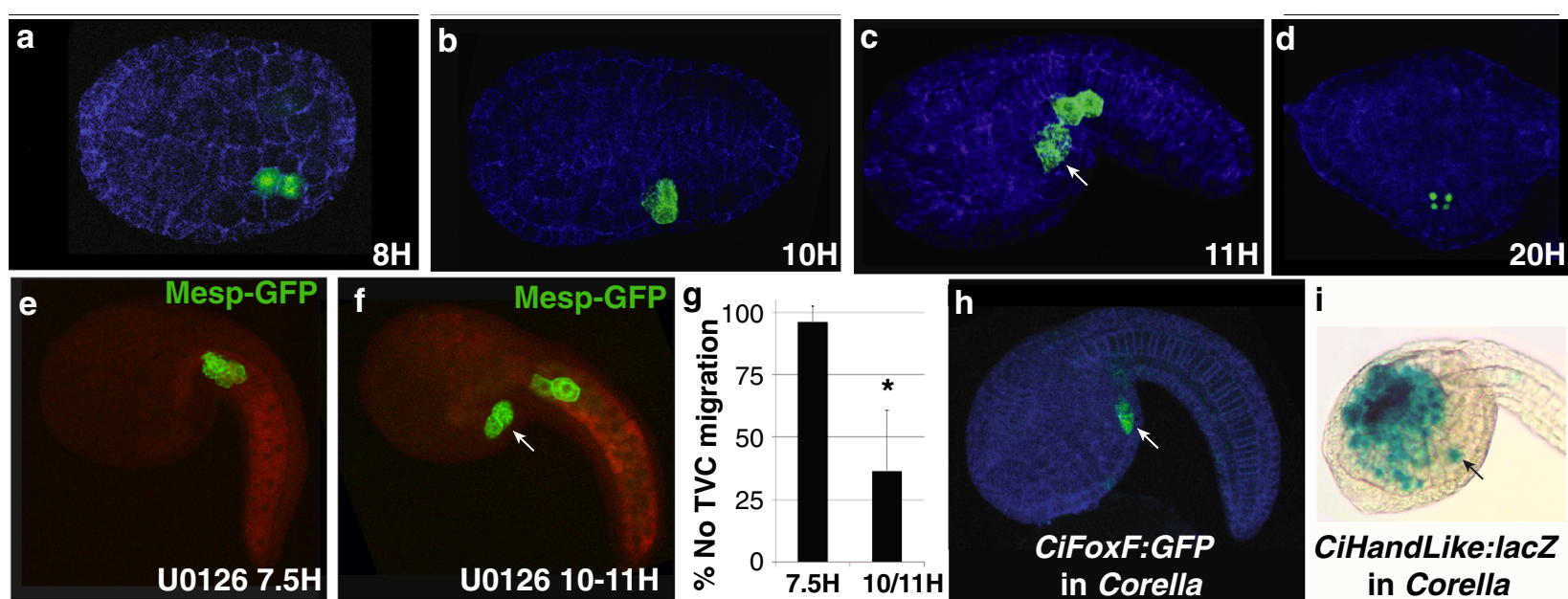

i

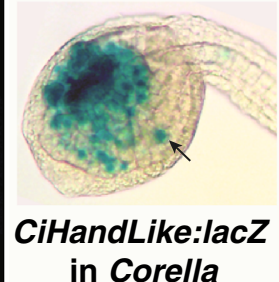

Fig. 2 Conserved founder cell lineage behavior and TVC induction in Corella embryos. a-c Representative Corella embryos expressing Cirobu.Mesp -1916:Esconsin-3XGFP in presumptive founder lineage cells. Note labeling of mitotic spindle in $8 \mathrm{H}$ embryo (a). Hours post-fertilization indicated at the lower right of each panel. $\mathbf{d}$ Representative Corella embryo expressing Cirobu.Mesp -1916:H2B:GFP to track founder lineage cell divisions in later stages. e, fTransgenic Cirobu.Mesp 1916:GFP Corella embryos treated with the Map Kinase inhibitor U0126 at 7.5 HPF, e immediately prior to founder cell division or $\mathbf{f} \sim 2 \mathrm{~h}$ after division at 10-11 HPF. Arrow points to migrated TVCs. $\mathbf{g}$ Summary of results for U0126 treatments. Data spans 6 trials, $N>70$ for each condition, Student's $T$ test, $p$ value $<0.0005$. Note that the levels of migration defects in the 10-1 1 HPF treatment samples were similar to basal levels seen in untreated, transgenic embryos (data not shown). h, i Representative embryos illustrating TVC expression for the Cirobu. FoxF-3052:GFP and Cirobu.Hand-like-2954/- 445:- 296:lacZ reporters

Comparative analysis of the C. robusta cardiopharyngeal GRN has been initiated in two species, Ciona savignyi and Molgula occidentalis. Regulatory elements and upstream trans-factors appear to be highly conserved in C. robusta and C. savignyi despite $\sim 100$ million years of rapid genomic divergence [29, 64]. In M. occidentalis and C. robusta, which diverged $\sim 390$ million years ago, cardiopharyngeal founder lineages still exhibit nearly identical patterns of cell division and transcription factor expression [10]. However, there have been partial or complete losses of intelligibility between cardiopharyngeal cis-regulatory elements in these two species, indicating that significant developmental systems drift has occurred both in cis and in trans [10].

To explore how evolutionary constraints influence drift in developmental programs, we have begun comparative studies of the cardiopharyngeal GRN in Corella inflata, a phlebobranch that diverged from C. robusta $\sim 270$ million years ago (Fig. 1d) (DeBiasse et al. 2019, in prep) [43]. C. inflata is experimentally tractable, as synchronized C. inflata embryos can be electroporated en masse to test reporter constructs, and we recently sequenced its genome and transcriptome (DeBiasse et al. 2019, in prep). We used this genome to characterize enhancers for key genes in the cardiopharyngeal GRN, including Mesp, FoxF, and Hand-like. We show that the trans-regulatory architecture of the cardiopharyngeal GRN is largely conserved between $C$. robusta and C. inflata, but cis-regulatory elements within this GRN exhibit different levels of conservation. These differences correspond to different structural and functional constraints.

\section{Results}

\section{C. inflata and C. robusta share a conserved TVC specification program}

To initiate our analysis of the Corella cardiopharyngeal GRN, we tested the activity of a characterized C. robusta reporter construct for the heart founder lineage transcription factor, Mesp (Cirobu.Mesp1916:Ensconsin:3XGFP) [56]. Fortunately, electroporation protocols developed for C. robusta [30] were also effective for C. inflata embryos. As observed in Ciona, the Cirobu.Mesp enhancer drove robust activity in Corella B7.5 founder lineage cells, including both TVC and ATM lineages. The Ensconsin:GFP reporter labels microtubules [56, 65], allowing us to deploy this construct to track founder cell lineage position and division in developing C. inflata embryos. As seen previously in both Molgulid and Cionid species, bilateral pairs of C. inflata heart founder cells divide asymmetrically at the early neurula stage $(\sim 8 \mathrm{HPF})$ to produce the heart progenitor and anterior tail muscle lineages (Fig. 2a, b). Further analysis will be required to determine if this division is unequal and whether differential induction involves receptor localization as characterized in C. robusta [57]. During tailbud stages, $C$. inflata heart progenitors undergo 
a conserved anterior migration along the epidermis into the ventral trunk region (Fig. 2c), where they undergo an unequal cleavage to form smaller medial and larger lateral daughters (Fig. 2d). Whether this represents an asymmetric division to produce pharyngeal muscle and heart precursors as seen in C. robusta will require further analysis [62]. We also used the Cirobu.Mesp reporter to examine whether TVC specification (as marked by anterior migration) is dependent on FGF/MapK signaling. As seen previously in both C. robusta and Molgula occidentalis embryos, treatment with the MEK inhibitor U0126 just prior to B7.5 founder cell division (late gastrula stage) blocked induction of the heart progenitor lineage (as indicated by lack of TVC migration, Fig. 2e, g), while treatment at a later time point had no effect (Fig. 2f, g) $[10,55]$. We also began to examine conservation of the heart gene network downstream of FGF-dependent induction. In $C$. robusta, a small group of transcription factors including FoxF, Hand-like, and GATAa are upregulated directly downstream of FGF/MapK induction (Fig. 1) [41]. Through in situ hybridization in tailbud stage embryos, we found that $C$. inflata Fox $F$ is expressed in the trunk epidermis and TVCs, mirroring similar expression in C. robusta embryos at this stage (Fig. 3f). This initial analysis indicates that the program for trunk ventral cell specification and migration in $C$. inflata and C. robusta embryos has been conserved.

\section{C. robusta cardiac gene enhancers drive TVC reporter expression in C. inflata}

To further explore developmental systems drift in the cardiopharyngeal gene regulatory network, we began to perform cross-species testing of regulatory elements. Since $C$. inflata and C. robusta shared a common ancestor more recently than C. robusta and $M$. occidentalis (Fig. 1d) [43], we hypothesized that there would be conservation in the trans-regulatory architecture despite divergence of cis-regulatory elements. Based on this hypothesis, we expected the $C$. inflata and C. robusta cardiopharyngeal GRN enhancers to display mutual intelligibility in cross-species testing but not to align or exhibit similar binding site arrangements. Alternatively, it is possible that both cis-regulatory elements and transregulatory architecture have been conserved, as seen in comparisons between C. savignyi and C. robusta [29, $41,50,58]$, or that there has been divergence of both the cis-regulatory elements and trans-regulatory architecture, as seen in comparisons between $M$. occidentalis and $C$. robusta [10]. To begin exploring these hypotheses, we tested two well-characterized C. robusta TVC enhancers, Cirobu.FoxF-3052:GFP and Cirobu.HandLike-2954/-445:-296:lacZ, in Corella embryos. In C. robusta, both of these enhancer elements mediate TVC expression immediately after TVC induction and are co-regulated by Ets1/2 and an ATTA-binding co-factor $[41,58]$. As seen with the Cirobu.Mesp-1916 enhancer (Fig. 2a-f), both these reporters recapitulated characterized Ciona expression patterns in transgenic Corella embryos. The FoxF reporter drove expression in the TVCs and trunk epidermis (Fig. 2h) and the Hand-like reporter drove expression in the TVCs and trunk endoderm along with weak expression in the ATM lineage (Fig. 2i). The cross-species intelligibility of these three reporters indicates that TVC specification and migration in Corella and Ciona embryos rely on a conserved set of upstream trans-factors.

\section{The FoxF TVC enhancer is highly conserved between $C$. inflata and C. robusta}

To further explore drift of the FoxF-regulatory element, we attempted to identify a candidate orthologous enhancer in Corella using mVISTA multi-sequence alignment [66]. This alignment revealed a small region of sequence conservation in $C$. inflata at the position of the previously characterized C. robusta FoxF TVC enhancer (Fig. 3a) [58]. Strikingly, this 183 bp region contained a set of three conserved Ets $1 / 2$ and two conserved ATTAbinding motifs that precisely matched the number, spacing, and arrangement of the characterized binding sites in the orthologous Ciona FoxF enhancer, while intervening DNA was poorly conserved (Fig. 3b). Reporter constructs containing this conserved element in $C$. inflata were able to drive TVC-specific expression in both $C$. inflata (Fig. 3c) and C. robusta (Fig. 3d). Thus, cross-species testing demonstrated mutual intelligibility of a remarkably well-conserved FoxF TVC enhancer (Figs. 2h, 3c, d).

To further evaluate whether the conserved region upstream of Corella FoxF represented a functionally constrained regulatory element, we cloned a 146 bp fragment containing the full set of conserved binding motifs. We then fused this minimal region to a $255 \mathrm{bp}$ basal promoter that had no independent reporter expression (data not shown). The resulting construct (Coinfl. FoxF -547/-401::-255) drove reporter expression in Corella B7.5 lineage cells, including the TVCs and ATM precursors (Fig. 3e, g). We then individually knocked out the five conserved binding motifs in this minimal element through site-directed mutagenesis and visualized reporter expression in $C$. inflata embryos. While the disruption of the first Ets1/2 (E1) or first ATTA (A1) binding motifs significantly reduced TVC reporter expression, knocking out the other binding motifs had no discernible impact (Fig. 3g). These results mirrored the results from a similar analysis of the C. robusta FoxF TVC enhancer [41, 58] with the exception of the second Ets1/2 (E2)-binding motif which was required in the $C$. robusta enhancer 


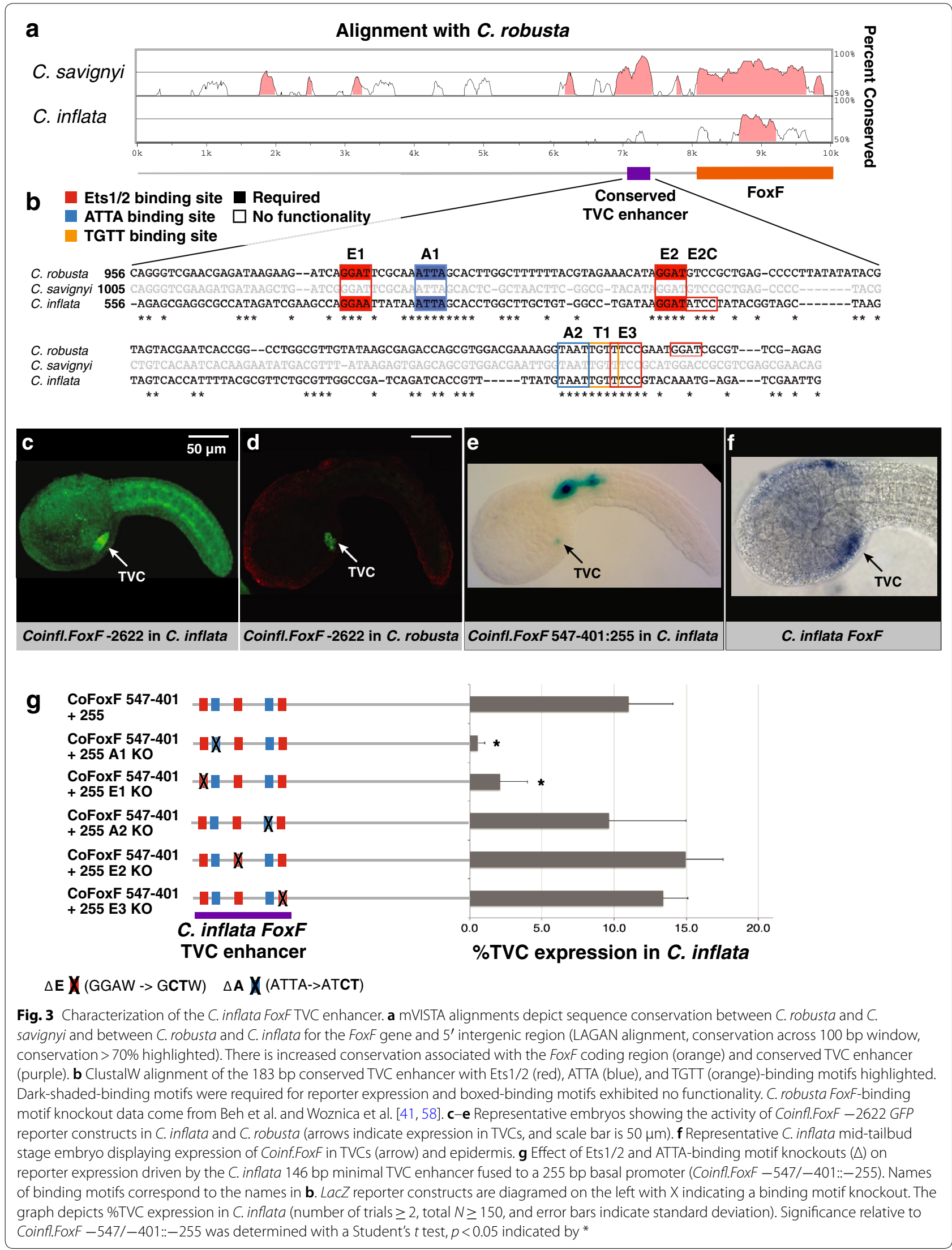




\begin{abstract}
(See figure on next page.)
Fig. 4 Characterization of the C. inflata Hand-like TVC enhancer. a Minimization of the C. inflata Hand-like (HL) upstream genomic fragment to test two predicted enhancers. LacZ reporter constructs are diagramed on the left. The graph depicts \%TVC expression in C. robusta (number of trials $\geq 2$, total $N \geq 75$, and error bars indicate standard deviation). Significance relative to Coinfl. $H L-1737$ was determined with a Student $t$ test $\left(p<0.001\right.$ indicated by $\left.{ }^{* *}\right)$. The second predicted enhancer is both necessary and sufficient for reporter expression in the TVCs. b-e Representative embryos showing the expression of $\mathrm{LaCZ}$ reporter constructs that contain the second predicted enhancer (Coinfl.HL -1048) or lack the second predicted enhancer (Coinfl.HL -899) in both C. robusta and C. inflata (arrows indicate expression in TVCs, and scale bar is $50 \mu \mathrm{m}$ ). $\mathbf{f}$ Effect of Ets $1 / 2$ and ATTA-binding motif knockouts $(\Delta)$ on the expression of a C. inflata Hand-like::LacZ reporter construct containing a 1048 bp upstream genomic fragment (Coinfl.HL -1048). Names of binding motifs correspond to the names in panel B. LacZ reporter constructs are diagramed on the left with $X$ indicating a binding motif knockout. The graph depicts \%TVC expression in C. robusta (number of trials $\geq 2$, total $N \geq 25$, and error bars indicate standard deviation). Significance relative to Coinfl.HL -1048 was determined with a Student $t$ test $(p<0.01$ indicated by ** and $p<.001$ indicated by ***). $\mathbf{g}$ Comparison of Hand-like TVC enhancer structure in C. robusta and C. inflata. Darkly shaded binding motifs were required for reporter expression. Lightly shaded binding motifs exhibited 'limited"functionality as assessed by mutagenesis of multiple sites in the minimal Cirobu. FoxF enhancer [41] or by a non-significant reduction in reporter expression following mutagenesis (this study). Boxed binding motifs exhibited no functionality. C. robusta Hand-like binding motif knockout data comes from Woznica et al. [41]
\end{abstract}

(Fig. 3g). This apparent divergence in enhancer structure may reflect the presence of a third (presumably supplemental) Ets1/2-binding motif in C. inflata immediately adjacent to the second Ets1/2 motif (E2C), potentially creating redundancy. These results suggest that selection has stringently constrained FoxF TVC enhancer structure, preventing any major shifts in the order, number, or spacing of binding sites over nearly 300 million years of rapid genomic divergence between $C$. robusta and $C$. inflata.

\section{Differential divergence of the Hand-like vs. FoxF TVC enhancer elements}

To determine if the rigorous conservation of the FoxF TVC enhancer was unique or reflected generally high levels of constraint in the cardiopharyngeal GRN, we characterized the C. inflata TVC enhancer for Hand-like. Hand-like and FoxF occupy very similar positions in the C. robusta cardiopharyngeal GRN [42]. Both these genes are expressed shortly after TVC induction. They are both regulated by Ets1/2 and an ATTA-binding co-factor and they encode key transcription factors for TVC progenitor fate (Fig. 1b). Based on the proposition that the hierarchical position of a gene within a GRN correlates with the level of selective constraint on its regulatory elements [4], we hypothesized that Hand-like and FoxF-regulatory elements would exhibit a similar level of conservation.

Sequence alignments did not reveal a conserved region in C. inflata associated with the characterized Handlike TVC enhancer in C. robusta (Additional file 1: Figure S1A) [66]. However, this analysis did not exclude the presence of a conserved enhancer that may have shifted position relative to the Hand-like gene and thus failed to align globally. We, therefore, searched more broadly for the C. inflata Hand-like TVC enhancer based on binding motif clustering and organization (see methods for further details). This approach identified two strong candidate elements in the $5^{\prime}$ intergenic region (Additional file 1: Figure S1B). The distal element (prediction 1) was located 1737-1587 bp upstream of the gene, in a similar position to the previously characterized $C$. robusta enhancer. The proximal element (prediction 2) was located 1048-898 bp upstream of the gene. Both predicted elements contained Ets $1 / 2$ and ATTA-binding motifs and exhibited some structural similarity to the previously characterized TVC enhancer of C. robusta Hand-like (Additional file 1: Figure S1B) [41].

We tested these computational predictions through sequential minimization of the $C$. inflata Hand-like $5^{\prime}$ intergenic region using $L a c Z$ reporter constructs (Fig. 4a). The full-length construct (Coinfl.HL -1737) containing both candidate elements had strong TVC expression in C. robusta, demonstrating that the Handlike TVC enhancer is intelligible by $C$. robusta. We employed C. robusta for further minimization experiments, because this species is more readily available than C. inflata. Deletions that removed the first candidate cis-regulatory element (Coinfl.HL -1615) or the region between the candidate cis-regulatory elements (Coinfl.HL -1048) did not affect TVC reporter expression (Fig. 4a, b), but removing the second candidate cis-regulatory element (Coinfl.HL -899) eliminated TVC reporter expression (Fig. 4a, c). A minimal 208 bp region encompassing the second candidate cis-regulatory element fused to a 299 bp basal promoter (Coinfl.HL -1048/-844::-299) drove strong TVC expression along with some ectopic expression in the mesenchyme, a hotspot for ectopic reporter expression [67], demonstrating that this region is both necessary and sufficient for Hand-like TVC expression (Fig. 4a). Coinfl.HL -1048 had strong TVC reporter expression (Fig. 4b) and Coinfl.HL -899 had no TVC reporter expression (Fig. 4c). Similar results were obtained in C. inflata (Fig. $4 \mathrm{~d}$, e). Thus, the Hand-like TVC enhancer is mutually intelligible in cross-species 


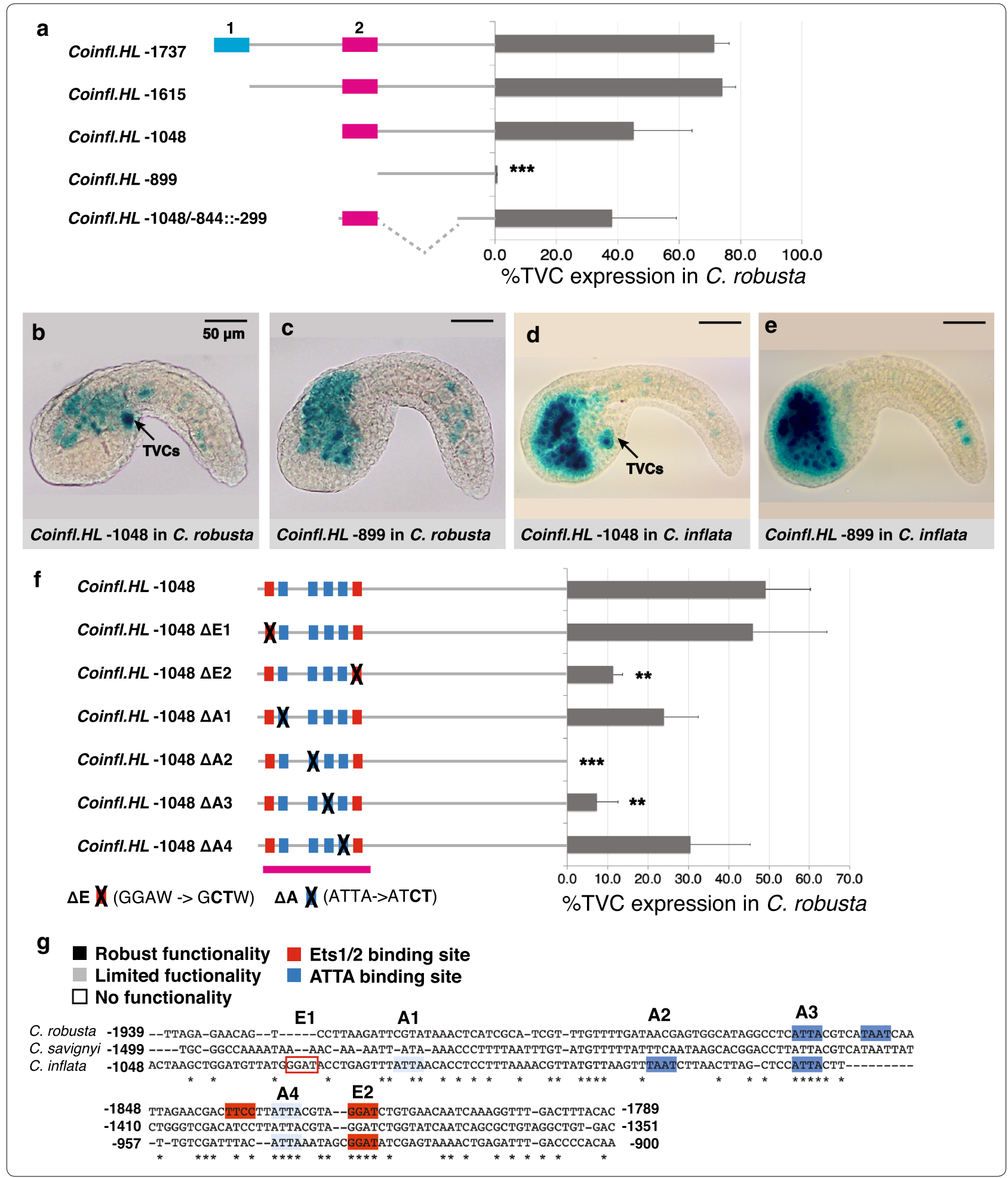

assays (Figs. 2i, 4c) while exhibiting substantially more divergence in binding motif organization in comparison with the FoxF TVC enhancer.
We next began to functionally characterize the binding sites in the C. inflata Hand-like TVC enhancer through site-directed mutagenesis (Fig. 4f). This enhancer contains two Ets1/2 and four ATTA-binding motifs (Fig. 4g). 
Knocking out the second or third ATTA motif (A2, A3) or the second Ets1/2 motif (E2) significantly reduced TVC reporter expression, while knocking out the remaining motifs did not significantly alter TVC reporter expression (Fig. 4f). In contrast, published mutational analysis of the C. robusta Hand-like element indicated that both Ets sites along with the first and second ATTA sites were required for full reporter activity (dark shading indicates functionally required binding motifs, Fig. $4 \mathrm{f}$ ) [41]. In summary, our analysis indicates that trans-regulation of Hand-like expression in the TVCs by Ets1/2 and an ATTA-binding co-factor has been conserved between these two species, while the cis-regulatory element has undergone substantial divergence, including changes in the number, order, orientation, and spacing of binding motifs. Thus, the cis-regulatory elements for FoxF and Hand-like appear to have experienced distinct levels of functional constraint, despite occupying similar positions in the cardiopharyngeal GRN.

\section{FoxF functions upstream of Hand-like in the cardiopharyngeal GRN}

When we aligned the FoxF and Hand-like TVC enhancers for C. robusta, C. savignyi, and C. inflata, we noticed a conserved TGTT-binding motif in both enhancers across all three species (Figs. 3b and Additional file 1: Figure S1B). TGTT is part of the consensus binding motif of Forkhead transcription factors such as FoxF (Additional file 1: Figure S2A) [15]. Prior studies noted the enrichment of this motif in Cionid TVC enhancer elements [41] and a recent study also detected a significant enrichment of putative FoxF-binding sites in the predicted cisregulatory elements of a wider range of primary TVC genes [68]. The conservation of this motif suggests that FoxF works to maintain its own expression and activate other primary TVC genes such as Hand-like in the C. robusta cardiopharyngeal GRN. As predicted by this hypothesis, mutation of the TGTT motif (T1) in the minimal C. robusta Hand-like TVC enhancer (Cirobu.HL -1914/-1314::-299) abrogated TVC reporter expression (Additional file 1: Figure S2B). In addition, mutation of the TGTT motif (T1) in the minimal C. robusta FoxF TVC enhancer (Cirobu.FoxF -1072/-847::pFkh) did not impact TVC expression, as predicted by the hypothetical role of this site in maintaining rather than initiating FoxF expression (Additional file 1: Figure S2B). Based on these results, we sought to determine if the TVC enhancer for GATAa also contains a conserved TGTT-binding motif. Using our script to computationally predict TVC enhancers for C. inflata GATAa, we identified one strong candidate element in the first intron (Additional file 1: Figure $\mathrm{S} 2 \mathrm{C}$ ), similar to the position of the characterized
C. robusta GATAa TVC enhancer [61]. A minimal 223 bp region of the intron containing this candidate element fused to a C. robusta Hand-like minimal promoter (Coinfl.GATAa +642/+820::Cirobu.Hand-like -299) was able to drive reporter expression in the TVCs (Additional file 1: Figure S3). Although the C. inflata GATAa enhancer diverged substantially from the $C$. robusta element, it still contains a conserved TGTT-binding motif (Additional file 1: Figure S2C). This finding suggests that GATA $a$ is also regulated by FoxF. Taken together, these results suggest that FoxF plays a central role in TVC specification, responding rapidly to FGF-dependent Ets1/2 activation, and contributing to the up-regulation of other primary TVC genes including Hand-like, while also maintaining its own expression. The putative role of FoxF upstream of Hand-like also suggests that the more stringent conservation of the FoxF-regulatory element may reflect this more critical functional role.

\section{Substantial divergence of the Mesp cardiopharyngeal founder cell enhancer}

To further investigate levels of drift across the cardiopharyngeal GRN, we characterized the regulatory element for founder cell expression of Mesp in C. inflata. In C. robusta, Mesp is expressed in the B7.5 cardiopharyngeal founder cell lineage downstream of TBX6b and LHX3 (Fig. 1) [50-53]. Sequence alignments did not reveal a conserved region in $C$. inflata associated with the characterized Mesp enhancer in C. robusta (Additional file 1: Figure S4A) [66]. We therefore computationally predicted candidate $C$. inflata Mesp enhancers based on binding site clustering. This approach yielded one candidate cis-regulatory element that aligned with the known cis-regulatory element for $C$. robusta (Additional file 1: Figure S4B) [51]. However, this candidate was a poor match, as it was missing the first two TBX6-binding motifs which were previously shown to be required in $C$. robusta [51]. We therefore started a sequential minimization analysis upstream of the candidate cis-regulatory element. The full-length construct (Coinfl.Mesp -866) drove strong expression in the founder lineage (ATMs and TVCs) in both C. inflata and C. robusta, demonstrating mutual intelligibility (Figs. 2, 5a, b, e). This reporter construct displayed almost no background expression (Fig. 5a, b, e). Two shorter constructs (Coinfl.Mesp -651 and Coinfl.Mesp -576) still drove strong expression in the founder lineage, but also produced ectopic expression in the primary trail muscle lineage (Fig. 5a, c). This result suggests that there is a silencer element 866$576 \mathrm{bp}$ upstream of Mesp that represses tail muscle lineage expression. A slightly shorter construct (Coinfl.Mesp -421 ) drove no expression in the founder lineages or primary trail muscle lineages (Fig. 5a, d), indicating that the 


\begin{abstract}
(See figure on next page.)
Fig. 5 Characterization of the C. inflata Mesp founder lineage enhancer. a Minimization of the C. inflata Mesp $5^{\prime}$ intergenic region to identify the B7.5 founder lineage enhancer. LacZ reporter constructs are diagramed on the left. The graphs depict \% founder lineage (TVC+ ATM) expression or \% founder lineage + primary tail muscle lineage expression in C. robusta and C. inflata (number of trials $\geq 2$, total $N \geq 25$, and error bars indicate standard deviation). Significance relative to Coinfl.Mes $p-651$ was determined with a Student $t$ test $(p<0.01$ indicated by ** and $p<0.001$ indicated by ${ }^{* *}$ ). b Representative C. robusta embryo showing founder lineage-specific expression of Coinfl.Mesp - 866 (arrows indicate TVCs and ATMs, and scale bar is $50 \mu \mathrm{m}$ ). c Representative C. robusta embryo showing the founder lineage and primary tail muscle lineage expression for Coinfl.Mesp -576. d Representative C. robusta embryo showing the lack of founder lineage expression for Coinfl.Mesp - 421. e Representative C. inflata embryo showing the founder lineage-specific expression for Coinfl.Mesp -866. f Effect of TBX6 and LHX3 binding motif knockouts ( $\triangle$ ) on the expression of the C. inflata Mesp founder cell enhancer. Binding motifs designated as shown in $\mathbf{g}$. LacZ reporter constructs are diagramed on the left with an $X$ indicating a binding motif knockout. The graphs depict \% founder lineage expression in C. robusta and C. inflata (number of trials $\geq 2$, total $N \geq 75$, and error bars indicate standard deviation). Significance relative to Coinfl.Mesp -576 or the minimal $-576 /-421$ enhancer was determined with a Student $t$ test $(p<0.05$ indicated by* and $p<0.01$ indicated by **). g Structure of the $C$. inflata Mesp founder cell enhancer. Darkly shaded green TBX6 motifs were required for reporter expression, and lightly shaded pink LHX binding motifs exhibited some functionality, as determined by mutagenesis of multiple motifs. There is no conservation of functional binding motifs in the aligned upstream genomic region of $C$. robusta. $\mathbf{h}$ Representative C. robusta embryo showing the founder lineage and primary tail muscle lineage expression for Coinfl.Mesp - 576/ $-421::-138$. i-k Representative C. robusta embryos showing lack of reporter expression for $\mathbf{i}$ Coinfl.Mesp $\Delta \mathrm{T} 1$, and $\mathbf{j}$ Coinfl.Mesp $\Delta \mathrm{L} 1,2,3,4$ and $\mathbf{k}$ reporter expression in the primary tail muscle lineage, but not the founder lineage for Coinfl.Mesp $\Delta L 4,5,6$
\end{abstract}

computationally predicted cis-regulatory element was not sufficient for reporter expression. Instead, we found that a region 576-421 bp upstream of Mesp fused to a 138 bp basal promoter (Coinfl.Mesp -576/-421::-138) drove strong founder lineage expression, demonstrating that this $155 \mathrm{bp}$ region is both necessary and sufficient for founder lineage expression (Fig. 5a). Strikingly, this $155 \mathrm{bp}$ minimal enhancer bears almost no sequence similarity to the characterized C. robusta element (Additional file 1: Figure S4C) and is also a very poor match to the globally aligned region 426-261 bp upstream of $C$. robusta Mesp (Fig. 5g) Thus, our analysis reveals substantial divergence between the minimal Mesp founder cell enhancers of these two species.

To begin investigating trans-regulation of Mesp in C. inflata, we mutagenized putative binding sites in the minimal reporter construct and assayed the impact on reporter expression in both $C$. robusta and $C$. inflata (Fig. 5f-k). The minimal C. inflata Mesp founder cell enhancer contains two TBX6-binding motifs and six LHX3-binding motifs (Fig. 5g). Knocking out either TBX6-binding motif (T1 or T2) completely eliminated founder lineage reporter expression in both $C$. robusta and C. inflata (Fig. 5f, i). In contrast, knocking out individual LHX3-binding motifs did not affect founder lineage reporter expression (data not shown). This result could reflect redundancy in the LHX3-binding sites, so we knocked out combinations of LHX3-binding motifs. When we knocked out the first four LHX3-binding motifs (L1, L2, L3, and L4), founder lineage and tail muscle lineage expression were lost in both C. robusta and $C$. inflata (Fig. 5f, j). When we knocked out the last three LHX3-binding motifs (L4, L5, and L6), founder lineage expression was almost completely eliminated, but primary tail muscle lineage expression was maintained
(Fig. 5f, k). Thus, trans-activation of Mesp by TBX6 and LHX3 appears to be conserved in C. inflata and $C$. robusta, while cis-regulatory elements have undergone substantial divergence.

In summary, our data indicate that upstream transcription factors dictating FoxF, Hand-like, and Mesp expression in the cardiopharyngeal GRN are conserved between $C$. robusta and C. inflata. However, the cisregulatory elements that control the expression of these genes exhibit distinct levels of conservation between $C$. robusta and $C$. inflata. The FoxF TVC enhancer is highly conserved, with identical organization of binding motifs, while the Hand-like and Mesp enhancers exhibit extensive divergence. These distinct levels of cis-regulatory conservation do not appear to reflect GRN hierarchy, as Mesp functions at the top of the GRN. Therefore, we began to explore alternative hypotheses regarding the exceptional conservation of the FoxF TVC enhancer over $\sim 270$ million years of rapid evolutionary divergence.

\section{Precise binding site spacing is required for FoxF TVC enhancer function}

There are a number of possible explanations for the relatively stringent conservation of the FoxF TVC enhancer between C. inflata and C. robusta. The first is that a specific organization of binding sites is required for physical interactions between transcription factors [3, 14]. Alternatively, the enhancer may be constrained to ensure precise temporal or spatial expression [69]. To distinguish between these hypotheses, we displaced the first Ets1/2binding motif (E1) in the C. robusta FoxF TVC enhancer and examined the impact on reporter expression. We chose this binding site because it is required for strong TVC expression in both C. robusta and C. inflata (Fig. 3b, g). Moreover, the ten base-pair spacing between this 


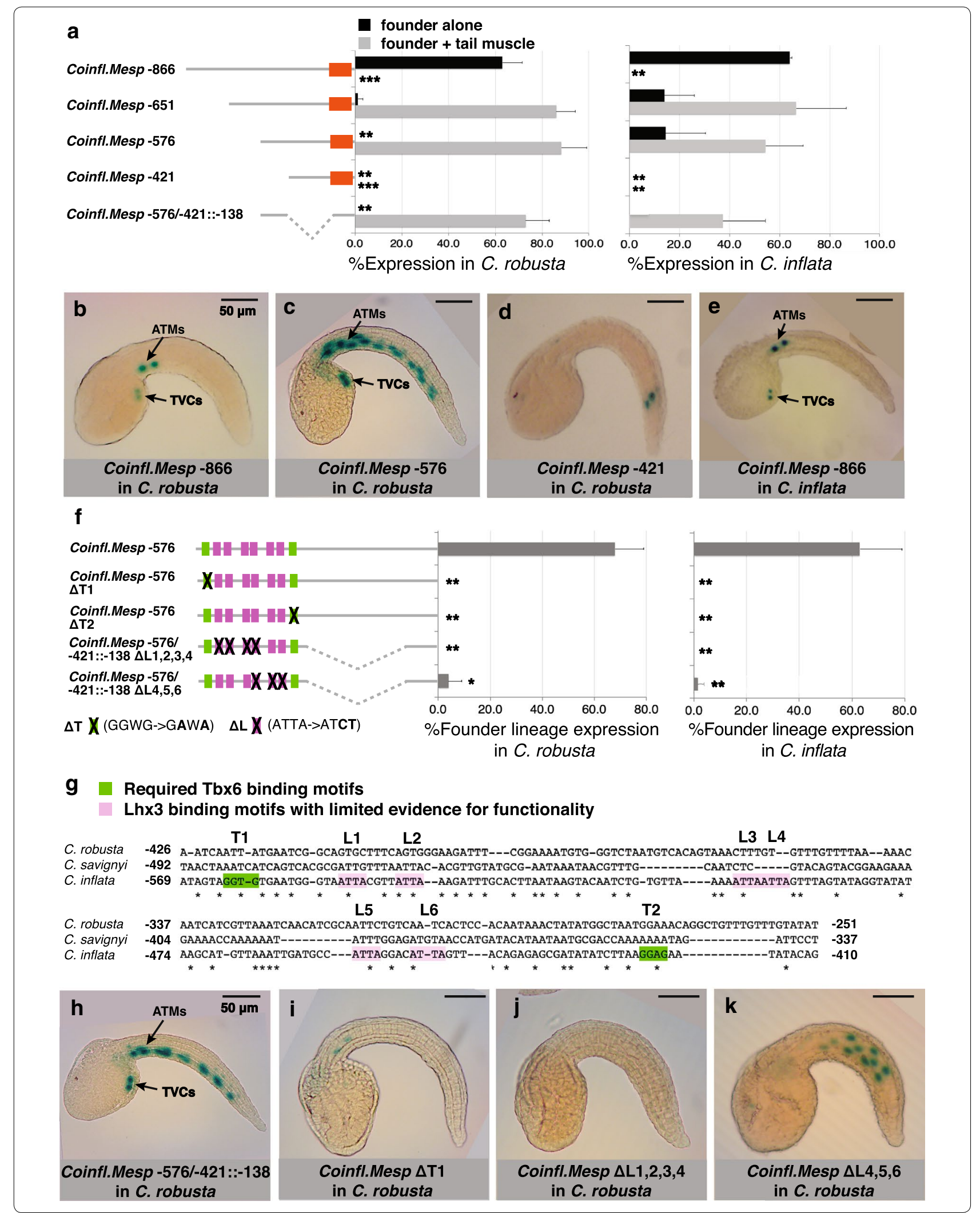



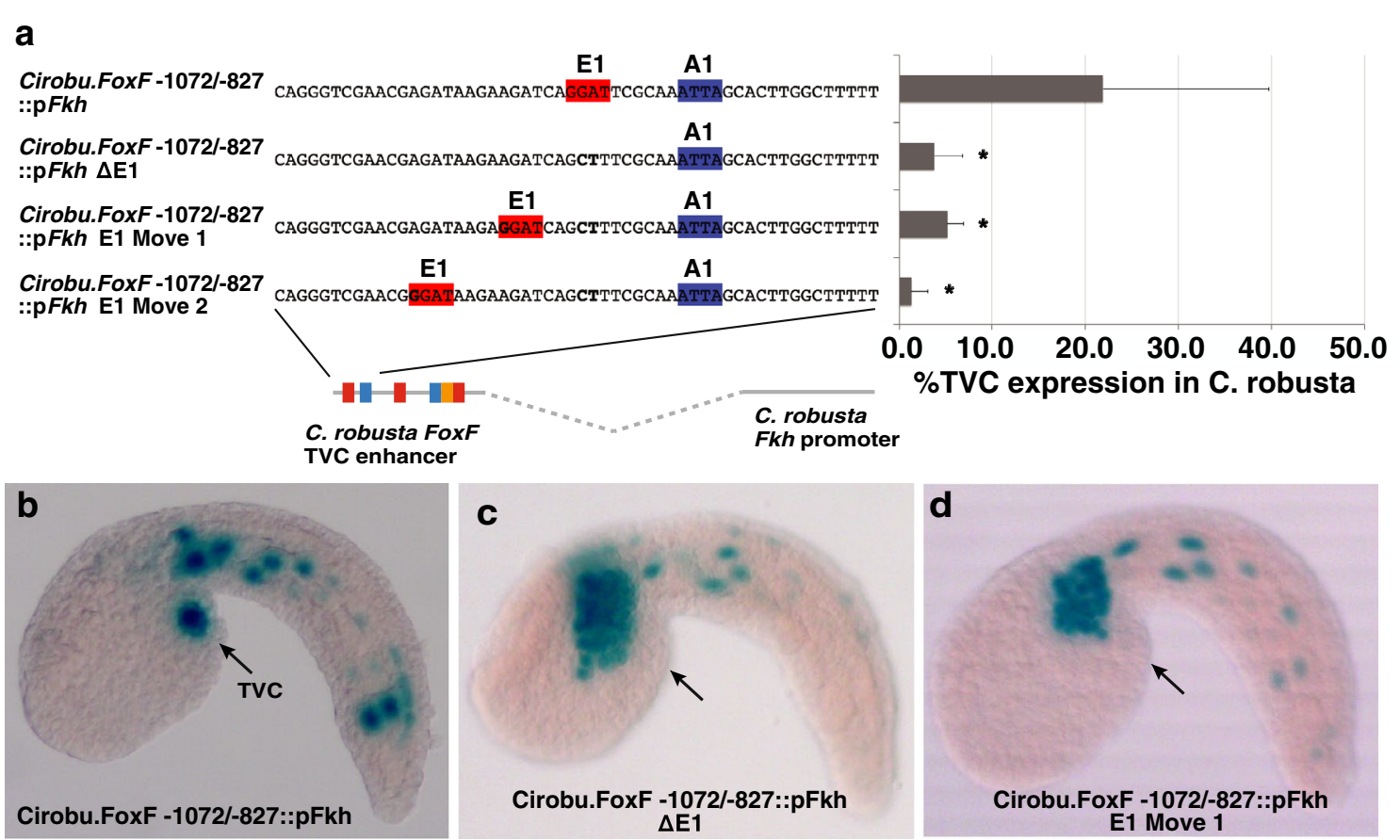

Fig. 6 Functional constraint on binding site spacing in the C. robusta FoxFTVC enhancer. a The first Ets1/2-binding site was moved by knocking out the endogenous binding site (GGAT $\longrightarrow$ GCTT) and introducing a new binding site using site-directed mutagenesis. Reporter constructs contained the 245 bp minimal C. robusta FoxF TVC enhancer fused to the C. robusta Fkh basal promoter (Cirobu.FoxF - 1072/-827::pFkh). The sequence of the enhancer region containing this first Ets1/2-binding site is shown on the left with Ets $1 / 2$ (red) and ATTA (blue)-binding sites highlighted. The graph depicts \%TVC expression in C. robusta (number of trials $\geq 2$, total $N \geq 75$, and error bars indicate standard deviation). b-d Representative C. robusta embryos showing reporter expression for b Corobu.FoxF - 1072/-827::pFkh, c Corobu.FoxF -1072/-827::pFkh $\triangle E 1$, or d Corobu.FoxF -1072/-827::pFkh Move 1. Arrows point to normal position of TVCs in the trunk region. Note substantial ectopic expression in the anterior tail muscle lineage (ATM) and in other muscle and mesenchyme lineage cells

binding motif (E1) and the first ATTA-binding motif (A1) is conserved between C. robusta and C. inflata. A ten base-pair increment between binding sites corresponds to a single helical turn and is often observed in enhanceosome-like cis-regulatory elements [14]. We displaced this first Ets1/2-binding site by knocking out the endogenous site and introducing a new site either 16 or 24 base pairs from the first ATTA site. We conducted this analysis in a $L a c Z$ reporter construct containing the minimal $245 \mathrm{bp}$ C. robusta FoxF TVC enhancer fused to the basal Forkhead promoter (Cirobu.FoxF -1072/-827::pFkh:lacZ). This is a slightly longer construct than the previously characterized 232 bp minimal reporter (Cirobu.FoxF -1072/-840::pFkh:lacZ) [58]. When the first Ets1/2binding motif (E1) was knocked out in the context of the $245 \mathrm{bp}$ minimal element, TVC reporter expression was significantly reduced (Fig. 6a, c). The introduction of new Ets1/2-binding sites 6 bp (Move 1), or 14 bp (Move 2) upstream of the original position failed to rescue TVC reporter expression (Fig. 6a, d). The fact that this reorganization reduced expression rather than altering temporal or spatial expression patterns supports the hypothesis that binding site organization is constrained by required interactions between trans-factors.

\section{Discussion \\ Developmental systems drift within the tunicate cardiopharyngeal GRN}

Mutual intelligibility in our cross-species assays suggests that the trans-regulatory architecture of the cardiopharyngeal GRN is largely conserved between $C$. inflata and $C$. robusta. These findings are in contrast to previous comparisons between $M$. occidentalis and C. robusta that revealed numerous instances of enhancer incompatibility caused by extensive trans drift in the cardiopharyngeal GRN [10]. Both these studies are based on functional analysis of minimal regulatory elements and thus may not encompass the full range of cis-regulatory function (as mentioned in the introduction, our use of the term drift in this instance and throughout the discussion is speculative, because observed changes in GRN structure may have undetected impacts on expression and thus may not be independent of selection). However, these studies still provide a robust framework for developing 
models regarding the rate and nature of developmental systems drift. In particular, these findings are congruent with two alternative models for the emergence of trans drift in developmental GRNs. Trans drift may arise at a steady rate, so that the amount of drift roughly correlates with the absolute evolutionary distance between two species and is not influenced by other taxonomic considerations. Alternatively, the rate of trans drift may vary due to factors independent of evolutionary distance. In particular, increased drift may occur during the divergence of major clades, such as that between phlebobranchs and stolidobranchs, in association with shifts in morphology or rewiring of underlying developmental gene networks. According to the first model, the differential occurrence of trans drift between $M$. occidentalis and C. robusta can be attributed to the longer period of divergence between these species, $\sim 390$ million years, in comparison with C. inflata, which diverged from C. robusta $\sim 270$ million years ago [43]. According to the second model, differential trans drift may have arisen during GRN rewiring associated with changes in body plan or divergence of developmental programs between Phlebobranchs and Stolidobranchs. A broader cross-species analysis is required to distinguish between these models.

Our analysis of the Mesp founder cell enhancer also provides an alternative perspective on differential divergence between trans-regulatory inputs [70]. The activation of Mesp by TBX6b is conserved between $M$. occidentalis, C. inflata, and C. robusta, while its activation by LHX3 is only conserved between $C$. inflata and C. robusta. Our results suggest that differential levels of constraint on these trans-factor inputs reflect a primary directive role for TBX6b, while LHX3 plays a more secondary, permissive role. When we removed the $300 \mathrm{bp}$ genomic region upstream of the C. inflata Mesp founder cell enhancer, we observed ectopic primary tail muscle lineage reporter expression. A similar result has been observed during deletion analysis of the C. robusta Mesp enhancer (Brad Davidson, unpublished results). Ectopic tail muscle expression is likely caused by TBX6b, which is expressed in a broad domain encompassing the B7.5 founder cells and neighboring tail muscle lineages [53]. According to this model, regions' upstream of the minimal Mesp element may contain a silencer bound by a tail muscle specific repressor. Thus, in tail muscle lineages, TBX6 may be able to activate Mesp expression independently of LHX3, which is expressed only in the endoderm/founder lineage cells. We are unsure why one set of LHX3 binding motif knockouts eliminated primary tail muscle and founder lineage expression, while another set only eliminated founder lineage expression. It is possible that mutagenesis of the first four LHX3-binding motifs accidentally impacted the binding motif of an additional transcription factor required for Mesp activation. Overall, our results provide preliminary support for the hypothesis that heterogeneous levels of constraint on trans-regulatory inputs reflect directive rather than permissive functional contributions. Clearly, further analysis is required to solidify our understanding of Mesp regulation and further test this general hypothesis.

Our findings provide more robust insights into cisregulatory drift. Sequence alignments and functional enhancer analysis reveal highly variable levels of divergence for cis-regulatory elements within the cardiopharyngeal GRN. The minimal FoxF TVC enhancer is highly conserved, with identical organization and spacing of binding motifs. In contrast, the minimal Handlike TVC enhancer is poorly conserved and the minimal Mesp founder cell lacks any apparent structural conservation. These findings do not align with models in which differential constraints associated with the position or function of a gene in a GRN dictate relative levels of cisregulatory drift. Rather, our findings suggest that drift is dictated by distinct structural and functional constraints that are unique to each cis-regulatory element. Our findings have also begun to illuminate the specific structural and functional constraints that dictate conservation of the FoxF enhancer, as discussed in the following section.

\section{Model for the constraints on the FoxF TVC enhancer}

Highly conserved enhancers generally reflect cooperative, position-specific interactions between bound transcription factors [14]. This type of highly conserved enhancer is known as an enhanceosome and is distinguished by conservation of the number, order, orientation, and spacing of binding motifs $[3,14]$. The prototypical enhanceosome is the interferon- $\beta$ cis-regulatory element [71]. Although relatively rare, additional enhanceosome-like cis-regulatory elements have subsequently been characterized [14, 17-19, 72]. However, general principles regarding the deployment of enhanceosomes within developmental GRNs have not been delineated. Mutations that disrupt the relative position of binding sites generally disable enhanceosome elements, presumably because they disrupt protein-protein interactions [16]. We show that displacing the first Ets1/2-binding motif in the C. robusta FoxF TVC enhancer significantly reduces reporter expression. This result suggests that the FoxF TVC enhancer is an enhanceosome-like cis-regulatory element, in which Ets1/2, the ATTA-binding co-factor, and possibly other proteins must physically interact to activate FoxF expression. However, further experimentation will be required to provide more definitive support for this hypothesis. In particular, the use of a wider range of mutations will help determine whether the specific mutations we introduced had unintended impacts, such 


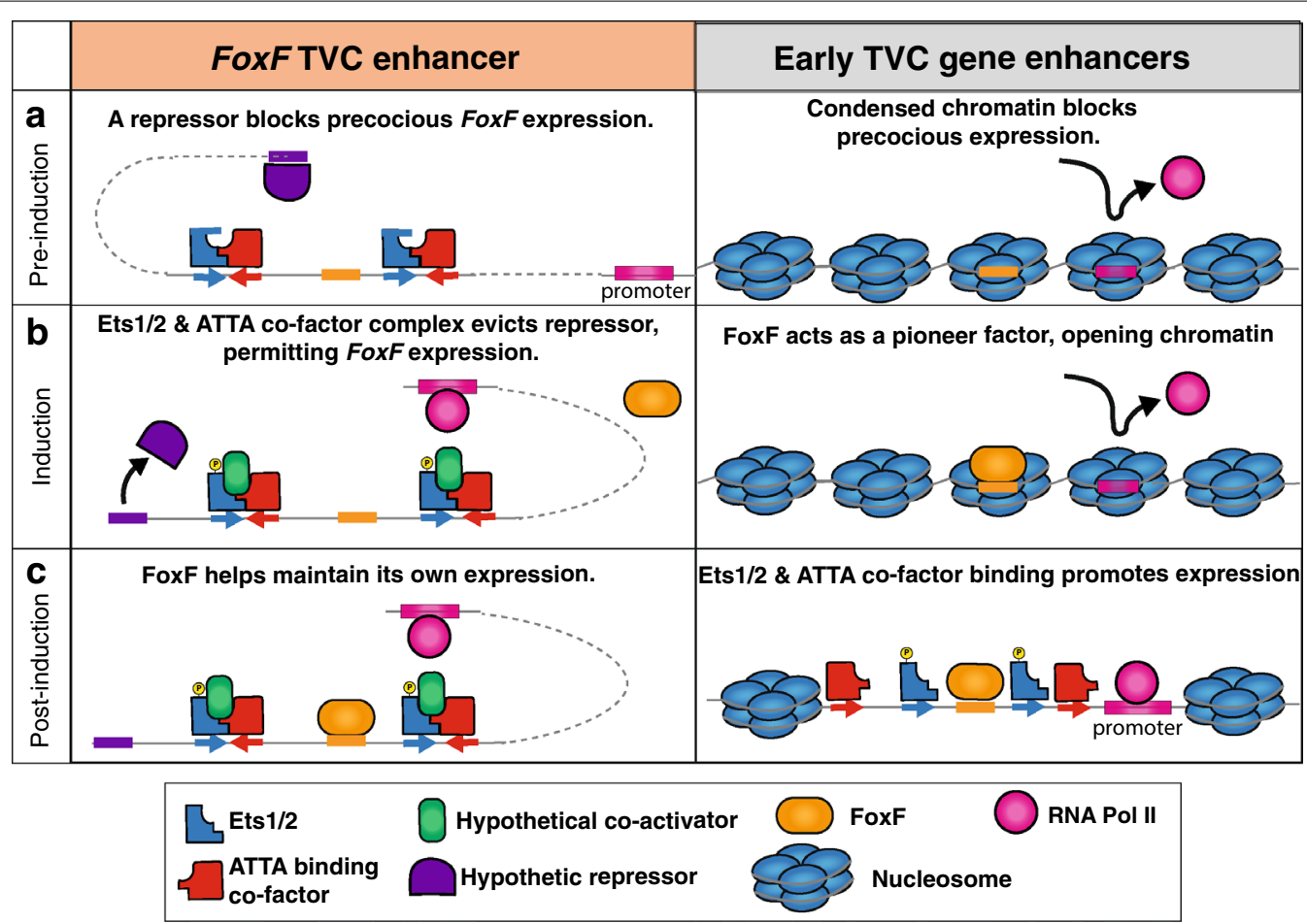

Fig. 7 Model for the differential constraint on FoxF vs. other early TVC enhancers. a Before FGF induction, the chromatin around early TVC gene enhancers is condensed preventing aberrant expression. In contrast, chromatin is decondensed at the FoxFTVC enhancer locus, suggesting that a repressor (purple) is required to prevent precocious expression. b FGF/MapK-signaling phosphorylates Ets 1/2 in the TVCs, permitting recruitment of a co-factor (green) that serves to lift repression. The cooperative recruitment of this co-factor constrains binding site position and orientation. FoxF (orange) then accumulates in the TVC nuclei, where it acts as a pioneer factor opening the chromatin around other TVC enhancers. c Once early TVC gene enhancers are open, the binding of Ets1/2, ATTA, and FoxF activates transcription in a non-cooperative fashion, as reflected by a lack of constraint on binding site position. FoxF also binds the FoxFTVC enhancer helping to maintain its own expression

as the creation or elimination of cryptic binding sites. In addition, by further varying binding site displacement, we can test whether presumed cooperativity is dependent on relative position on the helix. Furthermore, it will be interesting to analyze whether the conserved distances between other binding motifs in the FoxF minimal enhance also reflect functional constraints.

The deployment of an enhanceosome for regulation of FoxF may be associated with its role as a pioneer factor. This hypothesis arises from the recent findings of Racioppi et al., who found that FoxF promotes TVC specification by changing chromatin accessibility [68]. In particular, the binding of FoxF to the enhancers of other early TVC genes, including Hand-like and GATAa, appears to increase the accessibility of these cis-regulatory elements by decondensing chromatin, thereby enabling activation of these genes by Ets $1 / 2$, and the ATTA-binding co-factor [68]. Racioppi et al. also showed that CRISPR/Cas9 knockdown of FoxF led to down-regulation of several early TVC genes, including Hand-like [68]. Our mutational analysis of the FoxF-binding motif in the C. robusta Hand-like and FoxF TVC enhancer further supports the hypothesis that FoxF acts as a pioneer factor during TVC specification and also suggests that FoxF maintains its own expression.

\section{Conclusion}

Taken together, these results allow us to formulate a model that explains the specific deployment of a highly constrained, enhanceosome-like element for the regulation of FoxF (Fig. 7). Before FGF induction, the chromatin around the enhancers of most early TVC genes is condensed, which prevents aberrant expression (Fig. 7a). One exception is the FoxF enhancer, which remains decondensed, so it can mediate a rapid, primary response to FGF/MapK-dependent activation of Ets1/2 (Fig. 7a). Since chromatin condensation does not constrain aberrant expression of FoxF, another mechanism is required. We propose that this alternate mechanism involves the occupation of a silencer element located near the FoxF enhancer. Indeed, ectopic reporter expression throughout the B7.5 founder lineage in our 245 bp minimal FoxF enhancer construct (Fig. 6b) suggests that a silencer element serves to block precocious FoxF expression, 
possibly mediated by unphosphorylated Ets. According to our model, FGF/MapK-dependent phosphorylation of Ets1/2 leads to the formation of a complex with the ATTA-binding factor and the recruitment of a presumptive, non-DNA binding co-factor that is able to lift baseline repression (Fig. 7b). Once the FoxF gene is expressed, FoxF maintains its own expression and opens the chromatin around other TVC enhancers (Fig. 7c). This model may reflect a general principle for the seemingly sporadic occurrence of enhanceosomes. Namely, enhanceosomes may be specifically deployed for pioneer trans-factors, ensuring precise temporal or spatial expression despite a lack of chromatin-dependent regulation.

\section{Methods}

\section{Computational enhancer prediction}

The enhancers for C. inflata Hand-like, GATAa, and Mesp were computationally predicted based on structural similarity to the previously characterized enhancers in C. robusta [50, 51, 61]. A custom Python (version 2.7.13) script was used to slide a $150 \mathrm{bp}$ window over the $C$. inflata $5^{\prime}$ intergenomic region for each of these genes in 25 bp increments (https://github.com/colganwi/ CRMFinder). Each window position was scored with a linear combination of four features [1]: the number of oligomers $\geq 4$ bp which were present in both the window and the C. robusta enhancer, allowing for reverse complements, [2] similarity in oligomer ordering-the number of steps needed to transform one ordering into the other normalized by the number of conserved oligomers [3], similarity in enhancer position-the difference in the distance to the start codon normalized by the size of the $5^{\prime}$ intergenic region, and [4] the presence of specific conserved motifs, Ets1/2 (GGAW) for Hand-like and GATAa and TBX6 (GGNG) for Mesp.

\section{Molecular cloning LacZ reporter constructs}

Molecular cloning was performed according to established protocols [51]. C. inflata genomic regions used for enhancer analysis were amplified with sequence-specific primers carrying appropriate restriction sites (Additional file 1: Table S1). Cloning of C. robusta FoxF and Handlike minimal enhancers was described by Beh et al. and Woznica et al. [41, 58].

\section{Site-directed mutagenesis or insertion}

Sequence-specific primers containing desired point mutations or insertions (Additional file 1: Table S2) were used to generate sticky end fragment [51] or for whole plasmid amplification. For single-step whole plasmid amplification, we used mutagenesis primers between 30 and 60 bases in length, with a melting temperature $(\mathrm{Tm})$ of $\geq 78{ }^{\circ} \mathrm{C}$, the mutation placed in the exact center of the primer with 10-30 bp of correct sequence on both sides, and a minimum GC content of $40 \%$. Primers were diluted to $125 \mathrm{ng} / \mu \mathrm{l}$ and PCR run with 5-50 ng of template, Pfu ultra II taq polymerase (Agilent). If template was $>5 \mathrm{~kb}$, we added $3 \mu \mathrm{l}$ DMSO, and the reaction was run for 12-30 cycles based on the extent of the mutagenesis (12 for point mutations, 16 for 2-3 bp mutations, up to 30 for larger mutations). The PCR reaction was then cut with $1-2 \mu \mathrm{l}$ of $D p n \mathrm{I}$ at $37^{\circ} \mathrm{C}$ for $1 \mathrm{~h}$ and incubated at $70^{\circ} \mathrm{C}$ for 20 min prior to transformation of competent cells according to standard protocols.

\section{Embryological techniques Fertilization and dechorionation}

Adult $C$. inflata were harvested from docks on Lopez or San Juan Island, WA. M_REP (Carlsbad, CA) supplied adult $C$. robusta from multiple collection locations along the coast of San Diego, CA. C. robusta fertilization, dechorionation, electroporation, and staging were carried out as previously described [30, 56, 73]. For $C$. inflata, similar protocols were used with the following modifications. Sperm and then eggs were dissected from 4 to 6 gravid, freshly collected adults. Concentrated sperm from all adults was mixed in a $10 \mathrm{ml}$ dish of FNSW (filtered natural sea water). Eggs were dissected from each individual into a separate small dish of FNSW, and then, all eggs were rinsed once using $70 \mu \mathrm{m}$ mesh. Sperm was added to rinsed eggs, and after $12 \mathrm{~min}$, zygotes were passed through six rinse dishes. The zygotes were then transferred to a $10 \mathrm{ml}$ dish, and excess water was removed and replaced with a dechorionation solution $(10 \mathrm{ml} \mathrm{FNSW}+\mathrm{a} 200 \mu \mathrm{l}$ freshly thawed aliquot of $5 \%$ protease in FSW Streptomyces griseus, Sigma P8811-1G). After $4 \mathrm{~min}$, zygotes were pipetted gently and checked for dechorionation every minute. After 9-11 min, dechorionated zygotes were rinsed sequentially in six $10 \mathrm{ml}$ dishes of FNSW. Electroporation was as described for $C$. robusta except that only $50 \mu \mathrm{l}$ of total mannitol +DNA solution was used. Embryos were transfected with $100-300 \mu \mathrm{g}$ of DNA. Higher time constants $(\sim 20 \mathrm{~ms})$ appeared to give the best incorporation and did not hinder development. Embryos were cultured in gelatincoated dishes with $10 \mathrm{ml}$ of FNSW on a floating platform in a sea table $\left(\sim 14-16{ }^{\circ} \mathrm{C}\right)$ with the lids upside down to ensure that sea table water did not enter the cultures. Embryos were transferred after 2-4 h (4-16 cell stage) to a fresh dish of FNSW to ensure proper development. 


\section{$X$-gal staining}

Stage $22-23$ embryos were fixed with $0.175 \%$ glutaraldehyde and then stained with $\mathrm{X}$-gal to visualize $\mathrm{LacZ}$ reporter expression as previously described [51].

\section{Supplementary information}

Supplementary information accompanies this paper at https://doi. org/10.1186/s13227-019-0137-2.

Additional file 1. Additional figures and tables.

\section{Abbreviations}

GRNs: gene regulatory networks; TVCs: trunk ventral cells; FGF: fibroblast growth factor; MapK: Map Kinase; ATM: anterior tail muscle.

\section{Acknowledgements}

We wish to acknowledge Christina Cota for her technical assistance, mentoring, and training efforts throughout this project. We also wish to acknowledge Alberto Stolfi for his input on data analysis and interpretation.

\section{Authors' contributions}

Computational prediction of Ciinf.Mesp and Ciinf.Hand-like enhancer elements along with the design and implementation of cross-species testing of these elements was primarily conducted by WC. WC also designed and conducted the FoxF motif and enhancer spacing experiments and wrote the manuscript. $\mathrm{BD}$ conceived and oversaw much of the experimental design and conducted some of the experiments. JFR and MBD assembled the C. inflata genome and generated a revised phylogeny. AH helped to develop and refine protocols for transgenesis of C. inflata embryos along with conducting the Map Kinase inhibitor assays. AL conducted the initial analysis of the FoxF enhancer. IL and DR conducted the Ciinf.Mesp and Ciinf.Hand-like reporter assays in C. inflata, analyzed the resulting data, and helped generate relevant figures. The remaining student co-authors worked as a lab group associated with their Developmental Biology class (Bio24, 2017) to conduct Ciinf.Mesp and Ciinf. Hand-like reporter assays in C. robusta, analyze the resulting data and generate relevant figures. All authors read and approved the final manuscript.

\section{Funding}

Funding for BD was provided by the Swarthmore College Department of Biology and by NIH Grant Number R15HD080525-01. JFR and MBD acknowledge funding through the National Science Foundation under Grant Number 1542597.

\section{Availability of data and materials}

All data, scripts, sequences, and plasmid constructs will be made publicly available once the manuscript is accepted for publication.

\section{Ethics approval and consent to participate}

Not applicable.

\section{Consent for publication}

All contributors have seen and approved this manuscript.

\section{Competing interests}

The authors declare that they have no competing interests.

\footnotetext{
Author details

${ }^{1}$ Broad Institute, Cambridge, USA. ${ }^{2}$ Swarthmore College, Swarthmore, USA. ${ }^{3}$ Thomas Jefferson University Sidney Kimmel Medical College, Philadelphia, USA. ${ }^{4}$ University of North Carolina, Chapel Hill, USA. ${ }^{5}$ Whitney Laboratory for Marine Bioscience, St. Augustine, USA. ${ }^{6}$ University of Florida, Gainesville, USA. ${ }^{7}$ Icahn School of Medicine at Mount Sinai, New York, USA. ${ }^{8}$ Perelman School of Medicine, Philadelphia, USA. ${ }^{9}$ University of Colorado Boulder, Boulder, USA. ${ }^{10}$ Duke University School of Medicine, Durham, USA. ${ }^{11}$ Rothman Institute, Philadelphia, USA. ${ }^{12}$ University of Pittsburgh, Pittsburgh, USA. ${ }^{13}$ University of California San Francisco, San Francisco, USA. ${ }^{14}$ Memorial Sloan Kettering Cancer Center, New York, USA.
}

Received: 3 June 2019 Accepted: 13 September 2019

Published online: 11 October 2019

\section{References}

1. Levine M, Davidson EH. Gene regulatory networks for development. Proc Natl Acad Sci USA. 2005;102(14):4936-42.

2. Davidson EH, Levine MS. Properties of developmental gene regulatory networks. Proc Natl Acad Sci USA. 2008;105(51):20063-6.

3. Spitz F, Furlong EE. Transcription factors: from enhancer binding to developmental control. Nat Rev Genet. 2012;13(9):613-26.

4. Peter IS, Davidson EH. Evolution of gene regulatory networks controlling body plan development. Cell. 2011;144(6):970-85.

5. Halfon MS. Perspectives on gene regulatory network evolution. Trends Genet. 2017;33(7):436-47.

6. Wray GA. The evolutionary significance of cis-regulatory mutations. Nat Rev Genet. 2007:8(3):206-16.

7. Sucena E, Stern DL. Divergence of larval morphology between Drosophila sechellia and its sibling species caused by cis-regulatory evolution of ovo/ shaven-baby. Proc Natl Acad Sci USA. 2000;97(9):4530-4.

8. Gompel N, Prud'homme B, Wittkopp PJ, Kassner VA, Carroll SB. Chance caught on the wing: cis-regulatory evolution and the origin of pigment patterns in Drosophila. Nature. 2005;433(7025):481-7.

9. Combs PA, Fraser HB. Spatially varying cis-regulatory divergence in Drosophila embryos elucidates cis-regulatory logic. PLoS Genet. 2018;14(11):e1007631.

10. Stolfi A, Lowe EK, Racioppi C, Ristoratore F, Brown CT, Swalla BJ, et al. Divergent mechanisms regulate conserved cardiopharyngeal development and gene expression in distantly related ascidians. Elife. 2014;3:e03728.

11. Haag ES. The same but different: worms reveal the pervasiveness of developmental system drift. PLoS Genet. 2014;10(2):e1004150.

12. True JR, Haag ES. Developmental system drift and flexibility in evolutionary trajectories. Evol Dev. 2001;3(2):109-19.

13. Long HK, Prescott SL, Wysocka J. Ever-changing landscapes: transcriptional enhancers in development and evolution. Cell. 2016;167(5):1170-87.

14. Arnosti DN, Kulkarni MM. Transcriptional enhancers: intelligent enhanceosomes or flexible billboards? J Cell Biochem. 2005;94(5):890-8.

15. Khan A, Fornes O, Stigliani A, Gheorghe M, Castro-Mondragon JA, van der Lee R, et al. JASPAR 2018: update of the open-access database of transcription factor binding profiles and its web framework. Nucleic Acids Res. 2018;46(D1):D260-6.

16. Thanos D, Maniatis T. Virus induction of human IFN beta gene expression requires the assembly of an enhanceosome. Cell. 1995;83(7):1091-100.

17. Srivastava V, Dey I, Leung P, Chadee K. Prostaglandin E2 modulates IL-8 expression through formation of a multiprotein enhanceosome in human colonic epithelial cells. Eur J Immunol. 2012;42(4):912-23.

18. Fiedler M, Graeb M, Mieszczanek J, Rutherford TJ, Johnson CM, Bienz M. An ancient Pygo-dependent Wnt enhanceosome integrated by Chip/ LDB-SSDP. Elife. 2015;4:e09073.

19. van Tienen LM, Mieszczanek J, Fiedler M, Rutherford TJ, Bienz M. Constitutive scaffolding of multiple Wnt enhanceosome components by Legless/ BCL9. Elife. 2017;6:e20882.

20. Kiontke K, Barriere A, Kolotuev I, Podbilewicz B, Sommer R, Fitch DH, et al. Trends, stasis, and drift in the evolution of nematode vulva development. Curr Biol. 2007;17(22):1925-37.

21. Verster AJ, Ramani AK, McKay SJ, Fraser AG. Comparative RNAi screens in C. elegans and C. briggsae reveal the impact of developmental system drift on gene function. PLoS Genet. 2014;10(2):e1004077.

22. Maduro M, Pilgrim D. Conservation of function and expression of unc-119 from two Caenorhabditis species despite divergence of non-coding DNA. Gene. 1996;183(1-2):77-85.

23. Romano LA, Wray GA. Conservation of Endo 16 expression in sea urchins despite evolutionary divergence in both cis and trans-acting components of transcriptional regulation. Development. 2003;130(17):4187-99.

24. Oda-Ishii I, Bertrand V, Matsuo I, Lemaire P, Saiga H. Making very similar embryos with divergent genomes: conservation of regulatory mechanisms of Otx between the ascidians Halocynthia roretzi and Ciona intestinalis. Development. 2005;132(7):1663-74. 
25. Landry CR, Wittkopp PJ, Taubes CH, Ranz JM, Clark AG, Hartl DL. Compensatory cis-trans evolution and the dysregulation of gene expression in interspecific hybrids of Drosophila. Genetics. 2005;171(4):1813-22.

26. Takahashi H, Mitani Y, Satoh G, Satoh N. Evolutionary alterations of the minimal promoter for notochord-specific Brachyury expression in ascidian embryos. Development. 1999;126(17):3725-34.

27. Dehal P, Satou Y, Campbell RK, Chapman J, Degnan B, De Tomaso A, et al. The draft genome of Ciona intestinalis: insights into chordate and vertebrate origins. Science. 2002;298(5601):2157-67.

28. Delsuc F, Brinkmann H, Chourrout D, Philippe H. Tunicates and not cephalochordates are the closest living relatives of vertebrates. Nature. 2006;439(7079):965-8.

29. Johnson DS, Davidson B, Brown CD, Smith WC, Sidow A. Noncoding regulatory sequences of Ciona exhibit strong correspondence between evolutionary constraint and functional importance. Genome Res. 2004;14(12):2448-56.

30. Corbo JC, Levine M, Zeller RW. Characterization of a notochord-specific enhancer from the Brachyury promoter region of the ascidian, Ciona intestinalis. Development. 1997;124(3):589-602.

31. Khoueiry P, Rothbächer U, Ohtsuka Y, Daian F, Frangulian E, Roure A, et al. A cis-regulatory signature in ascidians and flies, independent of transcription factor binding sites. Curr Biol. 2010;20(9):792-802.

32. Cota CD, Segade F, Davidson B. Heart genetics in a small package, exploiting the condensed genome of Ciona intestinalis. Brief Funct Genomics. 2014;13(1):3-14.

33. Christiaen L, Wagner E, Shi W, Levine M. Electroporation of transgenic DNAs in the sea squirt Ciona. Cold Spring Harb Protoc. 2009;2009(12):pdb.prot5345

34. Swalla BJ. Building divergent body plans with similar genetic pathways. Heredity. 2006;97(3):235-43

35. Lemaire P. Unfolding a chordate developmental program, one cell at a time: invariant cell lineages, short-range inductions and evolutionary plasticity in ascidians. Dev Biol. 2009;332(1):48-60.

36. Kumano G, Nishida H. Ascidian embryonic development: an emerging model system for the study of cell fate specification in chordates. Dev Dyn. 2007;236(7):1732-47.

37. Lemaire P. Evolutionary crossroads in developmental biology: the tunicates. Development. 2011;138(11):2143-52.

38. Kocot KM, Tassia MG, Halanych KM, Swalla BJ. Phylogenomics offers resolution of major tunicate relationships. Mol Phylogenet Evol. 2018;121:166-73.

39. Nydam ML, Harrison RG. Polymorphism and divergence within the ascidian genus Ciona. Mol Phylogenet Evol. 2010;56(2):718-26.

40. Tsagkogeorga $\mathrm{G}$, Cahais $\mathrm{V}$, Galtier N. The population genomics of a fast evolver: high levels of diversity, functional constraint, and molecular adaptation in the tunicate Ciona intestinalis. Genome Biol Evol. 2012;4:852-61.

41. Woznica A, Haeussler M, Starobinska E, Jemmett J, Li Y, Mount D, et al. Initial deployment of the cardiogenic gene regulatory network in the basal chordate, Ciona intestinalis. Dev Biol. 2012;368(1):127-39.

42. Cota C, Palmquist K, Davidson B. Heart development in Ciona. Reference module in life sciences. Elsevier; 2017.

43. Delsuc F, Philippe H, Tsagkogeorga G, Simion P, Tilak MK, Turon X, et al. A phylogenomic framework and timescale for comparative studies of tunicates. BMC Biol. 2018;16(1):39.

44. Satou Y, Imai KS. Gene regulatory systems that control gene expression in the Ciona embryo. Proc Jpn Acad Ser B Phys Biol Sci. 2015;91 (2):33-51.

45. Roure A, Lemaire P, Darras $S$. An otx/nodal regulatory signature for posterior neural development in ascidians. PLoS Genet. 2014;10(8):e1004548.

46. Hudson C, Yasuo H. Similarity and diversity in mechanisms of muscle fate induction between ascidian species. Biol Cell. 2008;100(5):265-77.

47. Tokuoka M, Kumano G, Nishida H. FGF9/16/20 and Wnt-5alpha signals are involved in specification of secondary muscle fate in embryos of the ascidian, Halocynthia roretzi. Dev Genes Evol. 2007;217(7):515-27.

48. Evans Anderson $\mathrm{H}$, Christiaen L. Ciona as a simple chordate model for heart development and regeneration. J Cardiovasc Dev Dis. 2016;3(3):25.

49. Davidson B. Ciona intestinalis as a model for cardiac development. Semin Cell Dev Biol. 2007;18(1):16-26.

50. Davidson B, Levine M. Evolutionary origins of the vertebrate heart: specification of the cardiac lineage in Ciona intestinalis. Proc Natl Acad Sci USA. 2003;100(20):11469-73.

51. Davidson B, Shi W, Levine M. Uncoupling heart cell specification and migration in the simple chordate Ciona intestinalis. Development. 2005:132(21):4811-8.
52. Satou Y, Imai KS, Satoh N. The ascidian Mesp gene specifies heart precursor cells. Development. 2004;131(11):2533-41.

53. Christiaen L, Stolfi A, Davidson B, Levine M. Spatio-temporal intersection of $L$ hx 3 and Tbx6 defines the cardiac field through synergistic activation of Mesp. Dev Biol. 2009;328(2):552-60

54. Yagi K, Takatori N, Satou Y, Satoh N. Ci-Tbx6b and Ci-Tbx6c are key mediators of the maternal effect gene $\mathrm{Ci}$-macho1 in muscle cell differentiation in Ciona intestinalis embryos. Dev Biol. 2005;282(2):535-49.

55. Davidson B, Shi W, Beh J, Christiaen L, Levine M. FGF signaling delineates the cardiac progenitor field in the simple chordate, Ciona intestinalis. Genes Dev. 2006;20(19):2728-38.

56. Cooley J, Whitaker S, Sweeney S, Fraser S, Davidson B. Cytoskeletal polarity mediates localized induction of the heart progenitor lineage. Nat Cell Biol. 2011;13(8):952-7.

57. Cota CD, Davidson B. Mitotic membrane turnover coordinates differential induction of the heart progenitor lineage. Dev Cell. 2015;34(5):505-19.

58. Beh J, Shi W, Levine M, Davidson B, Christiaen L. FoxF is essential for FGF-induced migration of heart progenitor cells in the ascidian Ciona intestinalis. Development. 2007;134(18):3297-305.

59. Razy-Krajka F, Lam K, Wang W, Stolfi A, Joly M, Bonneau R, et al. Collier/ OLF/EBF-dependent transcriptional dynamics control pharyngeal muscle specification from primed cardiopharyngeal progenitors. Dev Cell. 2014;29(3):263-76.

60. Christiaen L, Stolfi A, Levine M. BMP signaling coordinates gene expression and cell migration during precardiac mesoderm development. Dev Biol. 2010;340(2):179-87.

61. Ragkousi K, Beh J, Sweeney S, Starobinska E, Davidson B. A single GATA factor plays discrete, lineage specific roles in ascidian heart development. Dev Biol. 2011;352(1):154-63.

62. Kaplan N, Razy-Krajka F, Christiaen L. Regulation and evolution of cardiopharyngeal cell identity and behavior: insights from simple chordates. Curr Opin Genet Dev. 2015;32:119-28.

63. Christiaen L, Davidson B, Kawashima T, Powell W, Nolla H, Vranizan K, et al. The transcription/migration interface in heart precursors of Ciona intestinalis. Science. 2008;320(5881):1349-52.

64. Small KS, Brudno M, Hill MM, Sidow A. A haplome alignment and reference sequence of the highly polymorphic Ciona savignyi genome. Genome Biol. 2007;8(3):R41.

65. Gallaud E, Caous R, Pascal A, Bazile F, Gagné JP, Huet S, et al. Ensconsin/ Map7 promotes microtubule growth and centrosome separation in Drosophila neural stem cells. J Cell Biol. 2014;204(7):1111-21.

66. Mayor C, Brudno M, Schwartz JR, Poliakov A, Rubin EM, Frazer KA, et al. VISTA: visualizing global DNA sequence alignments of arbitrary length. Bioinformatics. 2000;16(11):1046-7.

67. Harafuji N, Keys DN, Levine M. Genome-wide identification of tissuespecific enhancers in the Ciona tadpole. Proc Natl Acad Sci USA. 2002;99(10):6802-5.

68. Racioppi C, Wiechecki KA, Christiaen L. Combinatorial chromatin dynamics foster accurate cardiopharyngeal fate choices. bioRxiv. 2019.

69. Farley EK, Olson KM, Zhang W, Brandt AJ, Rokhsar DS, Levine MS. Suboptimization of developmental enhancers. Science. 2015;350(6258):325-8.

70. Dalal CK, Johnson AD. How transcription circuits explore alternative architectures while maintaining overall circuit output. Genes Dev. 2017;31(14):1397-405.

71. Maniatis T, Falvo JV, Kim TH, Kim TK, Lin CH, Parekh BS, et al. Structure and function of the interferon-beta enhanceosome. Cold Spring Harb Symp Quant Biol. 1998:63:609-20

72. Junion G, Spivakov M, Girardot C, Braun M, Gustafson EH, Birney E, et al. A transcription factor collective defines cardiac cell fate and reflects lineage history. Cell. 2012;148(3):473-86.

73. Hotta K, Mitsuhara K, Takahashi H, Inaba K, Oka K, Gojobori T, et al. A webbased interactive developmental table for the ascidian Ciona intestinalis, including 3D real-image embryo reconstructions: I. From fertilized egg to hatching larva. Dev Dyn. 2007;236(7):1790-805.

\section{Publisher's Note}

Springer Nature remains neutral with regard to jurisdictional claims in published maps and institutional affiliations. 